\title{
Chest pain in the accident and emergency department: is chest radiography worthwhile? worthwhile?
}

P. A. TEMPLETON, W. A. McCAllion, L. A. MCKINNEY, H. K. WILSON

Accident and Emergency Department, The Ulster Hospital, Dundonald, Belfast

\section{ABSTRACT SUMMARY}

Four per cent of patients attend the Accident and Emergency Department (A\&E) present with chest pain. In this prospective study of 297 patients the value of chest radiography is assessed. Overall, $23 \%$ of chest X-rays (CXRs) had an abnormality which influenced management of the patient, rising to $40 \%$ in those patients admitted to Coronary Care.

Twenty-nine per cent of CXRs were misinterpreted by Casualty Officers but resulted in the mismanagement of only six patients $(3.3 \%)$. Potentially serious errors were averted by early CXR audit by a Radiologist.

\section{INTRODUCTION}

One of the most common symptoms in patients presenting to A\&E is anterior chest pain. Junior doctors working in A\&E often lack experience in dealing with these patients, yet there is an obvious need to manage them correctly. Whilst electro-cardiography is a fundamental investigation in such circumstances (McCallion et al.), chest radiography is often performed, but there are conflicting views as to its efficacy (Buenger, 1988; Russell et al. 1988). In this prospective survey the value of routine chest radiography in patients presenting to A\&E with chest pain is assessed and the accuracy with which Casualty Officers interpret CXRs evaluated.

\section{METHODS}

In a prospective double blind study, 327 consecutive patients attended $A \& E$ in the Ulster Hospital, Dundonald, with chest pain during a 2-month period. Two hundred

Correspondence: L. A. McKinney FRCS, Accident and Emergency Department, The Ulster Hospital, Dundonald, Belfast BT16 ORH, Northern Ireland. 
and ninety seven had chest radiography. Of the remaining 30 patients, five were transferred to other hospitals and were not followed up, and 25 patients did not $\underset{\mathrm{\Phi}}{\overrightarrow{\mathrm{Q}}}$ have a CXR performed in A\&E. The Casualty Officers (three G.P. Trainees and five 0 Surgical S.H.Os) were asked to interpret the CXRs as 'normal' or 'abnormal' and, if $\frac{7}{0}$ 'abnormal', to state accurately the abnormality found. All CXRs were then validated by a Consultant or Registrar in Radiology who had access to the patients' clinical og details. Their opinion was then compared with that of the Casualty Officer. The clinical relevance of the CXR findings was assessed by a Senior Registrar in A\&E Medicine.

\section{RESULTS}

The total number of new patients attending A\&E during the 2-month study period was 7738 . The number of patients presenting with chest pain was $327(4 \%)$. There were 191 males and 136 females with an average age of 53 years (SD 15.4). A total of 103/327 patients were admitted: 65 males and 38 females, with an average age of 62 years (SD 13.6). The remaining 224 patients were discharged from A\&E.

Of the 297 CXRs performed, 183 were interpreted by the Casualty Officers. The remaining 114 CXRs were not commented upon in A\&E. In this group, 98 patients admitted to the Cardiac Unit had CXRs taken en route or immediately after admission to the ward to minimize the length of stay in A\&E, and 16 patients $\vec{\bullet}$ were discharged from A\&E without the Casualty Officer commenting on the CXR8.

Of the 297 CXRs validated, $166(56 \%)$ were normal and $131(44 \%)$ abnormal. The number of abnormalities and their clinical significance are shown in Table 1. I 131 abnormal CXRs there were 72 anomalies found in 68 X-rays which contributed to the patient's diagnosis or treatment. Of patients admitted to the Cardiac Unit, $39 / 98(40 \%)$ had a clinically relevant CXR abnormality.

Casualty Officers correctly interpreted 130/183 (71\%) CXRs: $97 / 108(90 \%)$ of normal CXRs and 35/75 (47\%) of abnormal CXRs were correctly read. Errors made by Casualty Officers and their clinical significance are shown in Tables 1 and 2 . There were 20 clinically relevant errors in CXR interpretation resulting in the mismanagement of six patients $(3.3 \%)$ : four major false negative and two minor false positive errors. Of the false negative errors, one patient diagnosed as having 'pleurisy' and discharged from A\&E, had radiological evidence of right lower lobe collapse and would have warranted further investigation; one patient with radiological evidence of pulmonary oedema was discharged but recalled on the basis of ECG and CXR abnormalities; and two patients with pneumathoraces were mis- $\frac{T}{0}$ diagnosed and discharged but recalled following CXR validation. Two CXRs were overdiagnosed as showing acute inflammatory changes where none existed and 0 both patients were treated inappropriately with antibiotics.

\section{DISCUSSION}

Chest pain is common, accounting for $4 \%$ of A\&E attenders in this study. Chest 


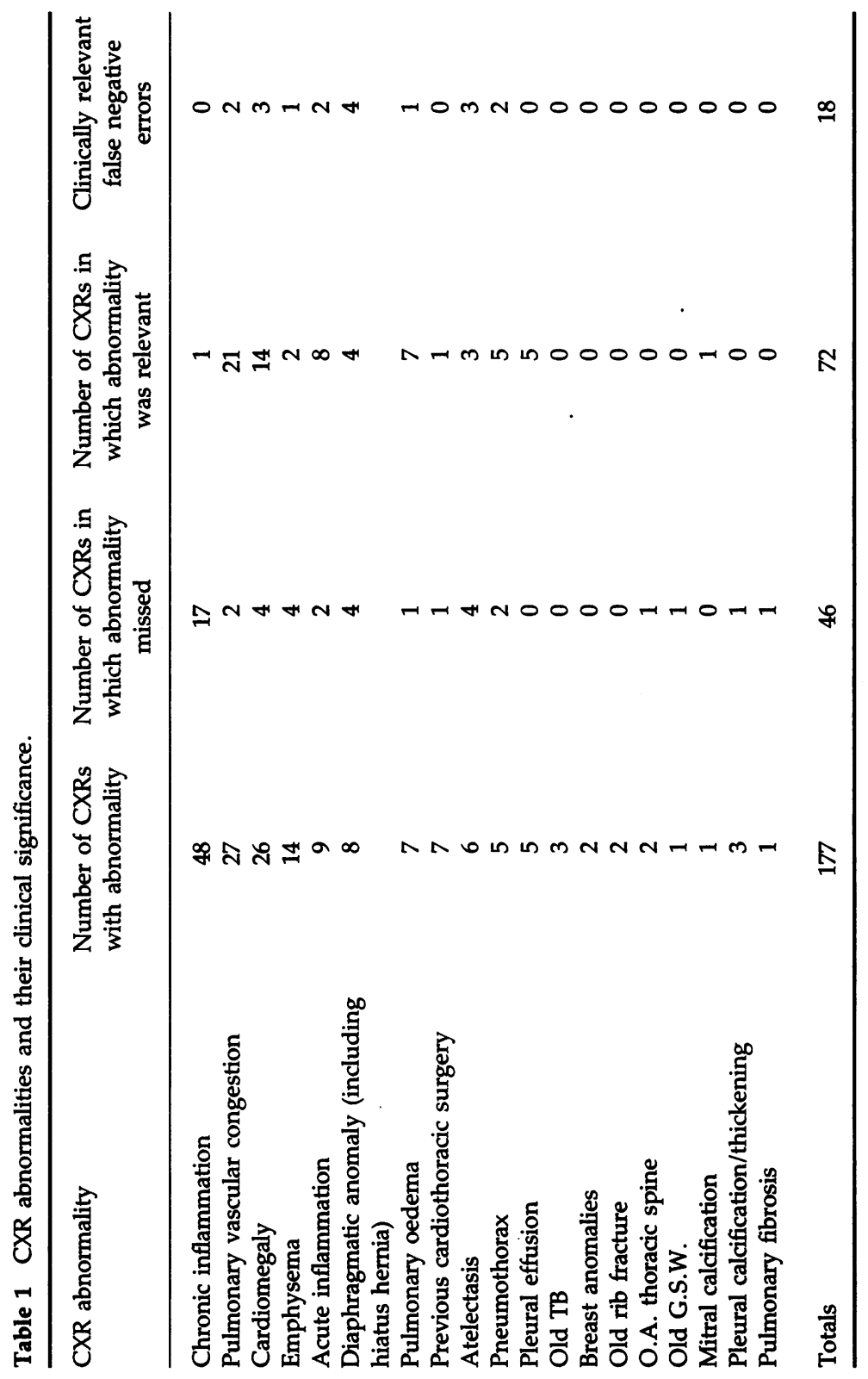


Table 2 False positive errors made in CXR interpretation and their significance.

\begin{tabular}{lcc}
\hline $\begin{array}{l}\text { CXR 'abnormality' } \\
\text { misdiagnosed by } \\
\text { Casualty Officer }\end{array}$ & $\begin{array}{c}\text { Number of CXRs with } \\
\text { this false positive } \\
\text { error }\end{array}$ & $\begin{array}{c}\text { False positive } \\
\text { errors resulting } \\
\text { in mismanagement }\end{array}$ \\
\hline $\begin{array}{l}\text { Acute inflammation } \\
\text { Chronic inflammation }\end{array}$ & 6 & 2 \\
Pulmonary vascular & 5 & 0 \\
congestion & 3 & 0 \\
Atelectasis & 1 & 0 \\
Cardiomegally & 1 & 0 \\
Total & 16 & 2 \\
\hline
\end{tabular}

radiography is often used routinely to evaluate these patients. To minimize mis- $\stackrel{\mathbb{D}}{3}$ management of patients and misuse of resources, it is important that investigations are appropriate and correctly interpreted. In one series of 5000 patients with chest 9 symptoms, 35\% had radiological evidence of 'serious disease' (Buenger, 1988), but $\mathrm{O}$ in a smaller series only $14 \%$ of patients with chest pain had a clinically relevant CXR abnormality (Russell et al., 1988). In this study 131/297 (44\%) CXRs were abnormal. Of these, 68 contained a clinically relevant abnormality which influenced $\stackrel{\oplus}{\circ}$ management of the patient, i.e. a clinically relevant pick-up rate of $23 \%$. This $\vec{\theta}$ figure rose to $40 \%$ in those patients admitted to the Cardiac Unit.

If patient mismanagement is to be minimized, it is essential that Casual Officers interpret CXRs accurately. Overall, $71 \%$ of CXRs were correctly interprete whereas only $47 \%$ of abnormal CXRs were correctly read. However, many of these errors were in relation to subtle and insignificant radiographic findings such as $\frac{2}{\circ}$ 'chronic inflammatory changes'. Twenty-eight per cent $(20 / 72)$ of all clinically $\overrightarrow{\vec{P}}$ relevant CXR abnormalities were missed, but resulted in the mismanagement of $\frac{\rho}{\Im}$ only six patients. However, due to regular audit of A\&E CRXs within $24 \mathrm{~h}$, and $\vec{F}$ due to the fact that a Consultant Radiologist was available during normal working hours if a second opinion was requested, all major false negative mismanagement errors were corrected.

\section{CONCLUSION}

In A\&E $96.7 \%$ of patients presenting with chest pain are managed correctly. $A \stackrel{7}{0}$ CXR is a useful investigation, particularly if a cardiac cause is suspected. Audit of all A\&E CXRs by a Radiologist within 24 hours, and continuing education of ${ }^{\circ}$ Casualty Officers in CXR interpretation, will further reduce patient mismanagement.

\section{ACKNOWLEDGEMENT}

We thank Mr. J. Shaw, Consultant in Accident and Emergency Department, for his 0 help and co-operation in preparing this manuscript. 


\section{REFERENCES}

Buenger R. E. (1988) Five Thousand Acute Care/Emergency Department Chest Radiographs: Comparison of requisitions with radiographic findings. Journal of Emergency Medicine 6, 197-202.

McCallion W. A., Templeton P. A., McKinney L. A. \& Higginson J. D. S. Missed Myocardial Ischaemia in Casualty: A need for ECG Audit. Archives of Emergency Medicine 8, 102-107.

Russell N. J., Pantin C. F. A., Emerson P. A. \& Crichton N. J. (1988) The Role of Chest Radiography in Patients Presenting with Anterior Chest Pain to the Accident and Emergency Department. Journal of the Royal Society of Medicine 81, 626-628. 